\title{
PARC Oat: A new Lodging-resistant, Late-maturing and High- yielding Variety of Oats (Avena sativa L.) for Pakistan
}

\author{
Muhammad Usama Hameed*, Zulfiqar Ali Gurmani, Sajjad Khan and Allah Bakhsh
}

Crops Sciences Institute, National Agricultural Research Center (NARC), Pakistan Agricultural Research Council Islamabad, Pakistan.

\begin{abstract}
PARC oat is a newly approved lodging-resistant, late-maturing, and high-yielding fodder oats variety developed at Fodder and Forage Research Program, National Agricultural Research Centre (NARC) Islamabad, Pakistan. This variety was selected from material imported from New Zealand for better agronomic characters and improved fodder yield. For initial two years (2006-07 and 2007-08) selections were made, and head-to-row method was used to reduce hetrozygosity. In 2009-10 and 20010-11, this line was evaluated in preliminary yield trials, where it produced average 80.71 tha $^{-1}$ green fodder yield while S-2000 produced 67.13 tha $^{-1}$ (16.82\% more green fodder yield than check). In terms of dry matter $20.100 \%$ increase in dry matter yield over check variety S-2000 was recorded (PARC Oat: 21.84 tha $^{-1,}$ S-2000 17.45 tha $^{-1}$ ). In National Uniform Fodder Yield Trials (NUFYT) for first year (during 2010-11) it produced average yield 46.71 tha $^{-1}$ while check variety S-2000 produced 43.45 tha $^{-1}$ (6.9\% Increase) at seven different agro-ecological locations. During 2011-12 and 2013-14 average yield was 47.065 tha $^{-1}$ but check (Sargodha, 2011) shows 42.76 tha $^{-1}$ (9.1\% increase). PARC-Oats exhibit a medium plant height $(110-120 \mathrm{~cm})$, high crude protein $(36.23 \%$ over check variety S-2000), thick stem (14.86\% more than check variety S-2000) and higher leaf area per plant (21.55\% over check variety S-2000). The variety is drought tolerant may be grown in semiarid and arid areas having minimum rainfall up to $300 \mathrm{~mm}$, highly lodging-resistant, late-maturing i.e. stay green till mid May, having up to $12 \%$ crude protein and having green fodder potential up to 100 tones ha $^{-1}$. PARC-Oat was approved by Variety Evaluation Committee (VEC) in March 2018.

Received | March 19, 2020; Accepted | June 23, 2020; Published | August 01, 2020

*Correspondence | Muhammad Usama Hameed, Crops Sciences Institute, National Agricultural Research Center (NARC), Pakistan Agricultural Research Council Islamabad, Pakistan; Email: uh_joiya@hotmail.com

Citation | Hameed, M.U., Z. Ali G, S. Khan and A. Bakhsh. 2020. PARC oat: A new lodging-resistant, late-maturing and high-yielding variety of oats (Avena sativa L.) for Pakistan. Pakistan Journal of Agricultural Research, 33(3): 601-608.

DOI | http://dx.doi.org/10.17582/journal.pjar/2020/33.3.601.608

Keywords | Fodder oat, Variety development, Pakistan
\end{abstract}

\section{Introduction}

$\mathrm{L}$ ivestock is an important industry in Pakistan. Its rural economy is largely dependent upon livestock sector as $35 \%$ income of 8 million families depends upon livestock. Being source of cash, food and fiber it provides people with most of their needs. With 191.3 million animal heads livestock contributes $60.54 \%$ to agricultural value addition and $11.22 \%$ to GDP
(GOP, 2018-19). To support livestock, nutrition of animals is of vital importance. In Pakistan main sources of nutrients for livestock are green fodder (51\%), crop residues (38\%), grazing (3\%), cereal byproducts $(6 \%)$ and oil cake meals (2\%) (Sarwar et al., 2002).

The estimated area under various fodder crops in the country is 2.03 million hectares with annual fodder 
production of 57 million tons (GOP, 2018-19). The average fodder production is $22.4 \mathrm{t} / \mathrm{ha}$ which is too low to meet even half of the maintenance ration of 201.9 million heads of livestock in the country (GOP, 201819). The reason is that inadequate importance has been given to fodder crops and as such very few varieties of fodder crops have been developed. Currently there is a great need for a new variety with increased potential to produce fodder and cope with climate change.

Oats (Avena sativa L.) is one of the most important Rabi crop used as forage, hay and silage both for irrigated and rain-fed areas in Pakistan. Its adaption in variable environments and soil types makes it better choice than other cereal crops (Ahmad et al., 2014). Oat provides green fodder during lean period in December and January, when there is a shortage of other fodders. Oats are now a very important winter fodder grown on small farms in Pakistan. Oats have changed from a minor fodder to a major crop, mainly because of the availability of improved cultivars and their ability to produce green feed during the midwinter lean period. (Hussain et al., 2004)

The existing oats varieties are low fodder yielding as they have lost their potential with passage of time due to diverse climatic conditions and are prone to damage by insect pest and diseases. Thus there is a dire need to develop improve fodder yield, nutritive, resistant to insect pest and diseases. The new oats variety "PARC Oats" is high yielding, and resistant to biotic and abiotic stresses. This paper will elaborate on its development.

\section{Materials and Methods}

Nine exotic lines of oats were introduced to Pakistan from Renewable Natural Resources Research Centre, Bajo, Wangdue-Bhutan during 2002. Initial evaluation of this germplasm during 2004-05 and 2005-06 revealed that despite being average in other morphological traits, a "NZ-0034" a genotype of New Zealand origin was found to be the top producer of green fodder and dry matter yield (94.68 and 25.51 tha $^{-1}$ respectively), it also had a large number of leaves and a good leaf to stem ratio. After selections adoptability trails of the said line have been conducted at four locations i.e. NARC Islamabad, FRI Sargodha, AARI Faisalabad, and BARI Chakwal. Preliminary yield trials (PYT) was conducted during 2009-10 and 2010-11. The candidate line along with check S-2000

was subjected to national uniform fodder yield trials (NUFYT) during 2010-11, 2011-12 and 2013-14. Performance on farmer field at four locations i.e. Begal Chakwal, Mial Fateh Jang, Sudazai Peshawar and Lateefwal Chakwal was performed during 2014-15. Agronomic trials were done by qualified agronomist and screening from disease and insects was done by Integrated Pest Management Program (IPMP) during 2017. Single adult aphids ( $R$. padi) were released in clip caged individually on a leaf of each variety. After 24 hours all aphids were removed except one 1st instar nymph which was left in the cage to mature. For each aphid the following data were recorded on daily basis; nymphal survival, time taken from birth to reproduction and daily reproduction of the adult over a 10 days period.

Disease incident study was done using following scales.

\section{Rating for smut/downy mildew \\ 1 = Highly Tolerant \\ $3=$ Tolerant \\ 5 = Moderately Tolerant \\ 7 = Susceptible \\ 9 = Highly Susceptible}

\section{Rating for leaf spot}

$1=$ Highly tolerant no pustule visible

3 = Tolerant $\quad$ few scattered pustules, usually seen after careful searching

$5=$ Moderately pustules common on leaves and eastolerant

$7=$ Susceptible ily observed but causing no apparent damage

pustules very common and damaging, few pustules on petioles and stems

9 = Highly suscep- ${ }^{-}$pustules very extensive on all plant parts, tible some death of leaves and other plant parts

\section{Statistical analysis}

The data were subjected to analysis of variance (ANOVA) and means were compared using least significant differences test (LSD) as proposed by Steel et al. (1997).

\section{Results and Discussion}

\section{Yield trials}

PARC Oats (NZ-0034) was planted along with 
a check variety S-2000 at four different locations during 2009-10 and 2010-11 for adaptation yield trials. Candidate line showed high green fodder yield at each location (Table 1) and in average produce $11.16 \%$ more yield than check. Highest yield was produced at NARC Islamabad $\left(80.71\right.$ tha $\left.^{-1}\right)$ followed by Faisalabad $\left(67.97\right.$ tha $\left.^{-1}\right)$ and the lowest yield was obtained at BARI Chakwal (33.75 tha $\left.{ }^{-1}\right)$. Table 1 shows the results of adaptation yield trials.

Results of preliminary yield trails clearly show the superiority of "PARC-Oats" over check (Table 2). In two years average increase in fodder yield over check S-2000 was $16.82 \%$ as PARC-Oats shows 80.71 tha $^{-1}$ as compare to check which produce 67.13 tha $^{-1}$. Similar was the case in dry matter yield which was 21.84 tha $^{-1}$ and 17.45 tha $^{-1}$ for PARC-Oats and S-2000 respectively. Nehvi et al. (2007) and Hussain et al. (2004) also showed similar results. PARC-oats was well adapted on farmer's field. During evaluation it was revealed that it produces $44.83 \%$ more fodder than check as shown in Table 3.

Table 1: Green fodder yield ( $t$ ha $\mathrm{a}^{-1}$ ) of PARC oat compared to check S-2000 in adaptation yield trials.

\begin{tabular}{|c|c|c|c|c|c|c|}
\hline \multirow[t]{2}{*}{$\begin{array}{l}\text { Line/ } \\
\text { Check }\end{array}$} & \multicolumn{5}{|c|}{$\begin{array}{l}\text { Green fodder yield }\left(\mathrm{t} \mathrm{ha}^{-1}\right) \text { in four locations (Av. of two years 2009-10 Aver-age } \\
\text { and 2010-11) }\end{array}$} & \multirow[t]{2}{*}{$\begin{array}{l}\text { \% Inc. }(+) \text { or Dec. } \\
(-) \text { over check }\end{array}$} \\
\hline & NARC Islamabad & FRI Sargodha & AARI Faisalabad & BARI Chakwal & & \\
\hline PARC-Oats (NZ-0034) & 80.71 & 60.22 & 67.97 & 33.75 & 60.66 & $11.16 \%$ \\
\hline S-2000 (Check) & 63.42 & 59.54 & 60.55 & 32.08 & 53.89 & - \\
\hline Table 2: Perfor & $f D A D C \cap$ & ne & ield $t$ & & & \\
\hline \multirow{2}{*}{ Lines/Check } & 2009-10 $\left(\right.$ tha $\left.^{-1}\right)$ & \multicolumn{2}{|c|}{$2010-11\left(\right.$ tha $\left.^{-1}\right)$} & \multicolumn{2}{|l|}{ Average } & \multirow{2}{*}{$\begin{array}{l}\text { \% Increase over } \\
\text { check (GFY) }\end{array}$} \\
\hline & GFY & GFY & DMY & GFY & DMY & \\
\hline PARC Oats (NZ-0034) & 64.20 & 97.22 & 26.35 & 80.71 & 21.84 & 16.82 \\
\hline S-2000 & 56.17 & 78.09 & 20.30 & 67.13 & 17.45 & \\
\hline
\end{tabular}

Table 3: Green fodder yield (t/ha) of PARC-Oats at farmers' fields.

$\begin{array}{lll}\text { Locations } & \text { PARC-Oats } & \text { S-2000(Check) } \\ \text { Begal Chakwal } & 40.7 & 20.11 \\ \text { Mial Fateh Jang } & 53.9 & 38.14 \\ \text { Sudazai Peshawar } & 69.14 & 31.10 \\ \text { Lateefwal Chakwal } & 37.2 & 21.50 \\ \text { Average } & 50.23 & 27.71 \\ \text { \% Inc. over check } & 44.83 & -\end{array}$

The performance of PARC-Oats was also evaluated in the National Uniform Fodder Yield Trials conducted by National Coordinator during Rabi 2010-11,201112 and 2013-14 at 7, 7 and 9 locations respectively throughout Pakistan. The fodder yield data of PARCOats in comparison with other lines and check is showed in Tables 4, 5 and 6. On average of three years PARC-Oats $\left(46.94\right.$ tha $^{-1}$ ) show $10.1 \%$ increase in green fodder yield over check S-2000 (42.23 tha $\left.{ }^{-1}\right)$ and Sargodha 2011 (42.76 tha ${ }^{-1}$ ). During 2010-11 on average data of seven locations, PARC-Oats out yielded all other lines in trial including the check variety S-2000. PARC-Oats produced 46.71 tha $^{-1}$ green fodder yields as check produced 43.45 tha ${ }^{-1}$ (Table 4). Yusuf et al. (2012) also evaluated millet line on farmer field to evaluate its yield potential.

During Rabi 2011-12 on average data of seven locations, PARC-Oats out yielded the check variety Sargodha oats 2011. PARC-Oats produced 38.89 tha ${ }^{-1}$ green fodder yields as check produced 38.35 tha $^{-1}$ (Table 5). Hussain et al. (2004) also showed similar results.

During Rabi 2013-14 on average data of nine locations, PARC-Oats out yielded the check variety Sargodha oats 2011. PARC-Oats produced 55.24 tha ${ }^{-1}$ green fodder yields as check produced 47.17 tha $^{-1}$ (Table 6). Hussain et al. (2004) also showed similar results.

As availability of good quality seed is an integral part of producing a good quality fodder, PARC-Oats was also tested for grain yield for two years during 201011 and 2013-14. In comparison with check S-2000 $(2597 \mathrm{Kg} / \mathrm{ha}), \mathrm{PARC}-\mathrm{O}$ ats produced $3.63 \%$ more seed yield $(2695 \mathrm{Kg} / \mathrm{ha})$. The results can be seen in Table 7 . 
Table 4: Green Fodder yield ( $t \mathrm{ha}^{-1}$ ) of national uniform fodder yield trials on Oats for Rabi 2010-11.

\section{Entry name}

\begin{tabular}{lllllllll} 
& & \multicolumn{4}{c}{ Locations } & \multicolumn{4}{c}{ Average } \\
& $\mathbf{1}$ & $\mathbf{2}$ & $\mathbf{3}$ & $\mathbf{4}$ & 5 & $\mathbf{6}$ & 7 & \\
NZ- 0034 (PARC oat) & 64.20 & 37.27 & 59.33 & 66.20 & 33.33 & 25.57 & 41.05 & 46.71 \\
No. 663 & 61.11 & 29.16 & 57.67 & 70.83 & 33.92 & 25.10 & 32.24 & 44.29 \\
SGD-1 & 52.47 & 40.07 & 44.33 & 59.25 & 42.59 & 17.20 & 32.41 & 41.19 \\
No. 75525 & 50.00 & 30.09 & 52.67 & 58.52 & 29.16 & 19.53 & 38.58 & 39.79 \\
Caliber & 53.71 & 40.36 & 45.00 & 60.18 & 37.03 & 20.27 & 38.89 & 42.21 \\
S-2000 & 52.47 & 25.46 & 66.00 & 66.48 & 34.12 & 18.90 & 40.74 & 43.45 \\
Ck-1 & 52.47 & 35.31 & 58.67 & 64.81 & 40.74 & 22.03 & 40.74 & 44.97 \\
F-411 & 56.17 & 27.39 & 40.00 & 73.22 & 36.01 & 19.73 & 39.51 & 41.72 \\
LSD 0.05\% & 5.90 & 6.90 & 4.56 & 7.38 & 4.35 & 3.77 & 6.05 & 2.10 \\
CV & 6.10 & 11.90 & 4.90 & 6.50 & 6.90 & 10.30 & 10.30 & 2.80
\end{tabular}

\section{Green fodder yield $\left(\mathrm{t} \mathrm{ha}^{-1}\right)$}

Locations

verage

1. NARC, Islamabad; 2. SBBARS, Sakrind; 3. Sorgdha; 4. AARI, Faisalabad; 5. ARI Tandojam; 6. BARI, Chakwal; 7. ESPU, Faroogabad; 8. Average of 7 sites.

Table 5: Green Fodder yield ( ha $^{-1}$ ) of National Uniform Fodder Yield Trials on Oats for Rabi 2011-12.

\section{Entry name}

\begin{tabular}{lllllllll}
\multicolumn{2}{l}{ Locations } & & & & \multicolumn{2}{c}{ Average } \\
& $\mathbf{1}$ & $\mathbf{2}$ & $\mathbf{3}$ & $\mathbf{4}$ & $\mathbf{5}$ & $\mathbf{6}$ & 7 & \\
Ck-1 & 32.69 & 39.47 & 21.57 & 23.15 & 74.69 & 42.13 & 40.73 & 39.20 \\
F-411 & 10.09 & 37.23 & 22.97 & 27.47 & 71.30 & 44.91 & 41.04 & 36.43 \\
Sargodha -2 & 12.31 & 45.00 & 26.10 & 28.40 & 82.41 & 43.52 & 43.20 & 40.13 \\
Domount & 10.14 & 40.40 & 28.00 & 22.53 & 69.44 & 44.91 & 39.19 & 36.37 \\
Sargodha Oats 2011 (Check) & 10.93 & 35.07 & 28.34 & 23.77 & 81.17 & 42.59 & 46.60 & 38.35 \\
Caliber & 12.31 & 43.30 & 29.43 & 25.93 & 72.22 & 42.13 & 40.43 & 37.96 \\
Fatua & 14.26 & 42.17 & 27.23 & 29.63 & 64.20 & 39.35 & 43.82 & 37.24 \\
NZ-0034 (PARC oat) & 10.65 & 46.07 & 22.77 & 30.56 & 70.68 & 44.91 & 46.59 & 38.89 \\
No. 663 & 17.13 & 54.97 & 30.53 & 37.65 & 75.00 & 38.43 & 41.97 & 42.24 \\
Canadian & 16.67 & 44.97 & 26.97 & 34.88 & 62.65 & 40.28 & 41.04 & 38.21 \\
NARC-Oat (Check) & 14.54 & 52.73 & 27.20 & 31.79 & 60.80 & 47.69 & 42.90 & 39.66 \\
LSD (0.05\%) & 19.72 & 9.49 & 1.84 & 10.43 & 7.62 & 6.77 & 7.20 & 3.41 \\
CV & 77.80 & 12.80 & 4.10 & 21.50 & 6.30 & 9.30 & 10.00 & 5.20
\end{tabular}

1. Juglot; 2. AZARI, Bahawalpur; 3. BARI, Chakwal; 4. ARI Tandojam; 5. FRI, Sargodha; 6. NARC, Islamabad; 7. AARI Faisalabad; 8. Average of 7 sites.

Hussain et al.(2004) also showed similar results. Ahmad et al. (2005), Bakhsh et al. (2005) and Hussain et al. (2010) also compared candidate line with commercial checks to find its yield potential.

\section{Quality traits}

Quality of fodder enormously effect milk and meat yields in animals. scarce supply of quality fodder is main cause of low productivity of milk and meat in animals (Patel et al., 2011). The good fodder should be high in crude protein $(\mathrm{CP})$, and low in crude fiber. Fodder yield and quality is greatly influenced by plant age and crude protein content, older crops produced more dry matter.

Table 8 shows the results of quality analysis in PARC Oats as compared to check variety S-2000 it is clear that all the quality components were higher in PARC-Oats except ether extract but PARC-Oats shows significantly higher crude protein percentage (11.92\%) than S-2000 (8.75\%). 
Table 6: Green fodder yield ( $\mathrm{ha} \mathrm{a}^{-1}$ ) of national uniform fodder yield trials on oats for Rabi 2013-14.

\begin{tabular}{|c|c|c|c|c|c|c|c|c|c|c|}
\hline \multirow[t]{3}{*}{ Entry } & \multicolumn{10}{|c|}{ Green fodder yield $\left(\mathrm{t} \mathrm{ha}^{-1}\right)$} \\
\hline & \multicolumn{9}{|c|}{ Locations } & \multirow[t]{2}{*}{ Average } \\
\hline & 1 & 2 & 3 & 4 & 5 & 6 & 7 & 8 & 9 & \\
\hline Sargodha-1 & 69.14 & 46.63 & 38.61 & 11.33 & 22.75 & 43.36 & 72.84 & 53.09 & 74.38 & 48.02 \\
\hline No-632 & 75.00 & 57.00 & 37.50 & 10.33 & 23.90 & 39.97 & 68.21 & 50.62 & 66.67 & 47.69 \\
\hline Domount & 79.63 & 54.03 & 38.22 & 15.33 & 23.30 & 46.14 & 69.14 & 56.17 & 68.83 & 50.09 \\
\hline Sgd-4 & 74.38 & 53.77 & 37.22 & 19.00 & 22.85 & 46.60 & 71.30 & 47.53 & 72.22 & 49.43 \\
\hline Hay & 75.62 & 61.37 & 36.39 & 18.33 & 26.50 & 36.88 & 70.06 & 51.85 & 72.42 & 49.94 \\
\hline $\mathrm{F}-411$ & 78.09 & 59.13 & 38.89 & 12.67 & 25.25 & 42.44 & 51.85 & 60.49 & 76.24 & 49.45 \\
\hline Sargodha-2011 (Check) & 74.38 & 54.77 & 45.27 & 9.67 & 26.55 & 41.05 & 54.63 & 53.09 & 65.12 & 47.17 \\
\hline Oats-Local (Quetta) & 75.31 & 53.10 & 38.33 & 13.00 & 24.70 & 41.67 & 64.81 & 64.20 & 75.31 & 50.05 \\
\hline Oats-Local (Peshawar) & 76.85 & 69.53 & 39.60 & 13.00 & 23.40 & 43.83 & 66.05 & 51.85 & 77.78 & 51.32 \\
\hline NARC-Oat (Check) & 89.20 & 56.83 & 36.78 & 13.33 & 31.75 & 41.67 & 68.83 & 58.64 & 78.08 & 52.79 \\
\hline NZ-0034 (PARC oat) & 97.22 & 60.67 & 41.94 & 18.33 & 29.00 & 38.27 & 69.75 & 61.11 & 80.86 & 55.24 \\
\hline No. 97009 & 99.38 & 58.40 & 39.44 & 15.67 & 24.10 & 42.28 & 70.06 & 55.56 & 81.17 & 54.01 \\
\hline Wintroo & 92.59 & 55.50 & 36.37 & 12.67 & 28.50 & 42.28 & 59.26 & 54.32 & 78.70 & 51.13 \\
\hline $\operatorname{LSD}(0.05)$ & 12.91 & NS & NS & 4.18 & 3.26 & NS & 6.27 & 8.47 & 11.13 & 3.07 \\
\hline CV (\%) & 9.4 & 14.6 & 15.0 & 17.7 & 7.6 & 12.9 & 5.60 & 9.10 & 8.9 & 3.60 \\
\hline
\end{tabular}

1. NARC Islamabad; 2. AZRI Babawalpur; 3. BARI Chakwal; 4. ARI Quetta; 5. MARC Gilgit; 6. Tarnab Peshawar; 7. AARI Faisalabad; 8. FRI Sargodha; 9. LPR Okara.

Table 7: Grain yield (kg/ha) of oats line PARC-Oats and S-2000 at NARC, Islamabad.

\begin{tabular}{lllll} 
Line/Variety & \multicolumn{2}{c}{ Grain yield Kgha ${ }^{-1}$} & \% increase over check \\
PARC-Oats (NZ-0034) & $2010-11$ & $\mathbf{2 0 1 3 - 1 4}$ & Mean & \\
S-2000 (Check) & 2650 & 2740 & 2695 & +03.63
\end{tabular}

Table 8: Quality analysis of PARC-Oats and S-2000.

$\begin{array}{llllll}\text { Line/Variety } & \text { Moisture (\%) } & \text { Crude protein(\%) } & \text { Crude fibre (\%) } & \text { Ash (\%) } & \text { Ether extract + NFE (\%) } \\ \text { PARC-Oats (NZ-0034) } & 9.35 & 11.92 & 29.11 & 12.18 & 49.50 \\ \text { S-2000 } & 5.67 & 8.75 & 28.58 & 11.48 & 51.07\end{array}$

\section{Yield contributing traits}

Different traits are reported to effect yield. Hussain et al. (2010), Ahmed et al. (2014) and Nehvi et al. (2007) reported the positive effect of plant height, number of leaves/tiller, number of tillers/plant, and leaf area o green fodder yield. PARC Oats was tested for yield contributing traits along with two commercial cultivars i.e. S-2000; results revealed that all the varieties were significantly different (Table 9). PARC-Oats showed medium plant height $(119.33 \mathrm{~cm})$ which in addition to good stem thickness $(0.85 \mathrm{~mm})$ contributes to its lodging resistance and ultimately yield. Its leaf area was and number of leaves were fairly higher (120.33 and $6.50 \mathrm{~cm}^{2}$ respectively) as compared to check S-2000 (99 and $5.67 \mathrm{~cm}^{2}$ respectively) which clearly shows it superiority in yield and palatability (Han et al., 2017).

Table 9: Fodder yield contributing characters of oats line PARC-Oats in comparison with check.

$\begin{array}{lllll}\text { Lines/Variety } & \begin{array}{l}\text { Plant } \\ \text { height }(\mathbf{c m})\end{array} & \begin{array}{l}\text { Leaves/ } \\ \text { tiller }\end{array} & \begin{array}{l}\text { Stem thick- } \\ \text { ness }(\mathbf{c m})\end{array} & \begin{array}{l}\text { Leaf area } \\ \left(\mathbf{c m}^{2}\right)\end{array} \\ \text { PARC-Oats } & 119.33 & 6.50 & 0.85 & 120.33 \\ \text { S-2000 (Check) } & 124.00 & 5.67 & 0.74 & 99.00\end{array}$

\section{Production technology}

Supply of fodder mainly depends on the time of sowing. The recommended sowing season for oats is from October to November. Sowing date can differ keeping in view the different factors; Number and 
type of animals; rainfall; availability of fallow land; and irrigation water supply. To find out optimum sowing date for timely available and economically feasible fodder, sowing date trials on PARC-Oats were conducted at Fodder Research program NARC Islamabad during Rabi 2014-15and 2015-16. Five different sowing dates (15 Sep, 01 Oct, 15 Oct, 01 Nov, and 15 Nov.) for two years shows significant differences. By comparing the means sowing on 1st October yield highest fodder yield $\left(85.86\right.$ tha $\left.^{-1}\right)$ followed by $15^{\text {th }}$ September $\left(83.44\right.$ tha $\left.^{-1}\right)$. Results are shown in Table 10.

Table 10: Average green fodder yield (t/ha) of oat line "PARC-Oats" under various sowing dates.

$\begin{array}{llll}\text { Sowing dates } & \text { Years } & & \text { Average } \\ & \text { 2014-15 } & \mathbf{2 0 1 5 - 1 6} & \\ \text { 15.Sep } & 91.67 & 75.22 & 83.445 \\ \text { 01.Oct. } & 82.87 & 88.85 & 85.86 \\ \text { 15 Oct } & 74.54 & 72.45 & 73.495 \\ \text { 01.Nov } & 68.52 & 61.89 & 65.205 \\ \text { 15.Nov } & 53.24 & 45.78 & 49.51 \\ \text { LSD (0.05) } & 91.67 & 41.66 & 66.665 \\ \text { CV (\%) } & 3.66 & 5.87 & \end{array}$

Seed Rate trials on PARC-Oats were conducted at Fodder Research program NARC Islamabad during Rabi 2014-15and 2015-16. Six different seed rates $(70,75,80,85,90$ and $100 \mathrm{Kg} / \mathrm{ha})$ for two years shows significant differences. By comparing the means highest fodder yield was produces by the seed rate of $85 \mathrm{~kg} \mathrm{ha}^{-1}\left(58.51 \mathrm{tha}^{-1}\right)$ followed by $90 \mathrm{kgha}^{-1}(53.99$ tha $\mathrm{a}^{-1}$ ) yield was poor on $100 \mathrm{~kg} \mathrm{ha}^{-1}\left(48.46 \mathrm{tha}^{-1}\right)$ due to lodging. Results are shown in Table 11.

Table 11: Average green fodder yield (t/ha) of oat line "PARC-Oats" under various seed rates.

\begin{tabular}{|c|c|c|c|}
\hline \multirow{2}{*}{$\begin{array}{l}\text { Seed rate treat- } \\
\text { ments } \\
(\mathrm{kg} / \mathrm{ha})\end{array}$} & \multicolumn{2}{|c|}{ Green fodder Yield $\left(\mathrm{tha}^{-1}\right)$} & \multirow[t]{2}{*}{ Average } \\
\hline & 2014-15 & 2015-16 & \\
\hline 70 & 52.78 & 50.48 & 51.63 \\
\hline 75 & 45.37 & 44.55 & 44.96 \\
\hline 80 & 46.30 & 57.91 & 52.105 \\
\hline 85 & 56.48 & 60.55 & 58.515 \\
\hline 90 & 52.78 & 55.21 & 53.995 \\
\hline 100 & 52.78 & 44.15 & 48.465 \\
\hline LSD (0.05) & 2.50 & 1.84 & \\
\hline CV (\%) & 2.53 & 1.81 & \\
\hline
\end{tabular}

Row spacing trials on PARC-Oats were conducted at Fodder Research program NARC Islamabad during Rabi 2014-15and 2015-16. Five different row spacing $(15,22,30,37$ and $45 \mathrm{~cm})$ shows significant differences for Rabi 2015-16 but was not significant for 2014-15. By comparing the means highest fodder yield was produces by the $22 \mathrm{~cm}$ apart sown oats $(56.5$ tha $\left.^{-1}\right)$ followed by $30 \mathrm{kgha}^{-1}\left(52.17\right.$ tha $\left.^{-1}\right)$. Results are shown in Table 12. Romitti et al. (2016) reported similar results for oats.

Table 12: Average green fodder yield ( $t / h a)$ of oat line "PARC-Oats" under various row spacing.

$\begin{array}{llll}\begin{array}{l}\text { Row spacing treat- } \\ \text { ments (cm) }\end{array} & \text { Years } & \text { Average } \\ 15 & \mathbf{2 0 1 4 - 1 5} & \mathbf{2 0 1 5 - 1 6} & \\ 22 & 50.00 & 48.56 & 49.28 \\ 30 & 52.78 & 60.22 & 56.5 \\ 37 & 51.85 & 52.50 & 52.175 \\ 45 & 50.00 & 45.36 & 47.68 \\ \text { LSD (0.05) } & 42.59 & 40.98 & 41.785 \\ \text { CV (\%) } & \text { NS } & 1.96 & 48.465 \\ & 6.51 & 1.74 & \end{array}$

Fertilizer requirement trials on PARC-Oats were conducted at Fodder Research program NARC Islamabad during Rabi 2014-15and 2015-16. Seven different combinations of nitrogen and phosphorus were used. All the treatments show significant differences for Rabi 2015-16 and 201415. By comparing the means highest fodder yield was produces $150-75 \mathrm{NP}$ ratio $\left(71.88\right.$ tha $\left.^{-1}\right)$ followed by 150-25 NP (61.87 tha $\left.{ }^{-1}\right)$. Results are shown in Table 13. Hussain et al. (2010) showed similar results in their study. Rashid et al. (2007) accomplished that along with recommended nitrogen fertilizer phosphorus plays a vital role in improving fodder yield and quality. High quality fodder results in increased digestibility ultimately improving milk yield and resistance against diseases in animals.

These trials revealed that newly approved oats variety PARC-Oats performs best when sown from $15^{\text {th }}$ September to $15^{\text {th }}$ October. Optimum seed rate for PARC-Oats can be $80-85 \mathrm{kgha}^{-1}$. Optimum plant population and good yield of fodder can be obtained using row spacing from $20-30 \mathrm{~cm}$ along with using $150 \mathrm{~kg}$ nitrogen and $75 \mathrm{~kg}$ phosphorus for a hector.

Insect pest incidence

To observe the effect of aphid (Rhopalosiphum padi) 
on fodder oats, three varieties/lines viz. PARC-Oats, PD2-LV65, and S-2000 were evaluated at Fodder Program, NARC during winter 2015-16. Cultures of aphid ( $R$. padi) were established on these varieties for studying development time and fecundity (nymphs produced in 10 days). Development and fecundity of R. padi were measured as components of antibiotic resistance.

Table 13: Average green fodder yield (t/ha) of oat line "PARC-Oats" under various fertilizer treatments.

$\begin{array}{llll}\begin{array}{l}\text { Fertilizer treatments } \\ \text { N-P kg/ha }\end{array} & \begin{array}{l}\text { Green fodder yield }(\mathbf{t} \\ \left.\mathbf{h a}^{-\mathbf{1}}\right)\end{array} & \begin{array}{l}\text { Average } \\ \text { 2014-15 }\end{array} & \mathbf{2 0 1 5 - 1 6} \\ \mathbf{1 0 0 - 2 5} & 44.19 & 75.61 & 59.9 \\ 50-50 & 37.33 & 64.11 & 50.72 \\ 50-25 & 35.65 & 59.29 & 47.47 \\ 50-75 & 35.19 & 63.11 & 49.15 \\ 150-25 & 40.89 & 82.86 & 61.875 \\ 150-75 & 55.72 & 88.04 & 71.88 \\ 175-50 & 42.35 & 74.69 & 58.52 \\ 00-00 & 20.33 & 35.65 & 27.99 \\ \text { LSD }(0.05) & 0.97 & 4.37 & \end{array}$

Table 14 indicates that oat line "PARC-Oats" was found the most aphid tolerant. Aphids (R. padi) on this line showed a longer development time (9.2 days) from birth to reproduction and lower fecundity (14 nymphs produced in 10 days) than all the other varieties/line of oat under test. Hussain et al. (2011) also presented similar results. In the light of above results, oats line "PARC-Oats" may be recommended for approval and release as a fodder variety.

Table 14: Development time (days) and Fecundity (No. of nymphs produced in 10 days) of Aphid (R.padi) reared on different oat varieties/lines.

$\begin{array}{lcl}\text { Name of line/variety } & \begin{array}{l}\text { Development } \\ \text { time (days) }\end{array} & \begin{array}{l}\text { Fecundity (over } \\ \text { 10 days) }\end{array} \\ \text { PARC-Oats } & 9.2 & 14.00 \\ \text { PD2-LV65 } & 8.5 & 19.00 \\ \text { S-2000 (Check) } & 8.0 & 25.00\end{array}$

\section{Disease incidence}

Various diseases incidence on the new oats line "PARC-Oats" was observed by the Plant Pathologist FRI Sargodha during 2015-16. The data collected on various diseases are presented in Table 15.
The results show that new oats line "PARC-Oats" was found tolerant to loose/covered smut, leaf spot and downy mildew diseases while check variety "S-2000" was less tolerant than all the other entries. Hussain et al. (2011) also presented similar results.

Table 15: Reaction of promising oat lines/varieties against smut, leaf spot and downy mildew (Rating Scale 1-9).

$\begin{array}{llll}\text { Line/Variety } & \begin{array}{l}\text { Loose/cov- } \\ \text { ered smut }\end{array} & \text { Leaf spot } & \begin{array}{l}\text { Downy } \\ \text { mildew }\end{array} \\ \text { PARC-Oats } & 0 & 1 & 3 \\ \text { PD }_{2}-L_{65} & 0 & 1 & 3 \\ \mathrm{~S}_{65} 000 & 3 & 3 & 5 \\ \text { PARC oat (NZ-0034) } & 0 & 1 & 3\end{array}$

\section{Conclusions and Recommendations}

PARC Oats is a newly approved oat variety which has many advantages over current oat varieties in Pakistan. The variety is drought tolerant may be grown in semiarid and arid areas having minimum rainfall up to $300 \mathrm{~mm}$, highly lodging resistant, late maturing i.e stay green till mid May, more nutritious having up to $12 \%$ crude protein higher than any other oat variety in Pakistan and producing more green fodder up to 100 tones ha ${ }^{-1}$.

\section{Acknowledgement}

The authors highly acknowledge support of Crops Diseases Research Institute, NARC, Federal Seed Certification and Registration Department, Fodder Research Institute Sargodha, Pakistan and National Coordinator Cereals for their support during course of varietal development. No potential conflict of interest is reported for said work.

\section{Author's Contribution}

Muhammad Usama Hameed: Overall Write-up, Data collection, Data entry and analysis.

Zulfiqar Ali Gurmani: Conceived the idea, Overall Management of the article.

Sajjad Khan:Wrote Abstract, Conclusion and working paper of variety.

Allah Buksh: Technical Input at every step.

\section{Conflict of interest}

The authors have declared no conflict of interest. 
References

Ahmad, M., Akhtar, L. Hussain, S.Z. Siddiqi, M. Hussain, A. Rashid, G. Hussain and M. Arshad. 2005. Development of a high yielding wheat variety" Bahawalpur-97" for Southern Punjab, Pakistan. Pak. J. Sci. Ind. Res., 48(1): 42.

Ahmad, M., Z.A. Dar and H. Mehfuza. 2014. A review on oat (Avena sativa L.) as a dualpurpose crop. Sci. Res. Essays, 9(4): 52-59. https://doi.org/10.5897/SRE2014.5820

Bakhsh, A., M. Arshad and S.M. Iqbal. 2005. Development of chickpea blight resistant variety (Dasht) using combination of bulk population and pedigree breeding method. Pak. J. Bot., 37(2): 325 .

GoP, 2019. Ministry of finance, economic advisor's wing, Islamabad. Economic Survey of Pakistan.

Han, O., P. TaeIl, P. HyungHo, P. KiHun, O. YoungJin, K. KeeJong and K. DeaWook. 2017. Jungmo2501, a winter oat (Avena sativa L.) cultivar of lodging tolerance, early-heading and high forage yield. Korean J.Breed.Sci.,49(2): 8086. https://doi.org/10.9787/KJBS.2017.49.2.80 Hussain, A., S. Khan, A. Bakhsh, M. Imran and M. Ansar. 2010. Variability in fodder production potential of exotic oats (Avena sativa) genotypes under irrigated conditions. J. Agric. Res., 48(1): 65-71.

Hussain, A., S. Khan and D. Mohammad. 2004. Clipping of oats at various intervals on herbage yield, forage quality and seed yield. Pak. J. Agric. Res. 18(1): 2004.

Hussain, A., S. Khan, M. Zahid, S. Shafiq, S. Safdar and Z.Ali.2011. A new high yielding oat variety for fodder in the rainfed potohar and irrigated areas of Pakistan. Sci. Technol. Dev. (Pakistan).

Nehvi, F.A., Wani, A. Shafiq, A. Hussain, M.I. Maqhdoomi, B.A. Allai, W. Yousuf and Z.A. Dar. 2007. Stability analysis for yield and yield related traits in fodder oats (Avena sativa L.). Asian J. Plant Sci., 6(4): 628-632. https://doi. org/10.3923/ajps.2007.628.632

Patel, K.V. and A.D. Patel. 2011. Extent of heterosis for green fodder yield and its components traits in sorghum [Sorghum bicolor (L.) Moench]. Int. J. Plant Sci., 6(2): 348-351.

Rashid, M., A.M. Ranjha, M. Waqas, A. Hannan, A. Bilal, A. Saeed and M. Zafar. 2007. Effect of $\mathrm{P}$ fertilization on yield and quality of oat (Avena sativa L.) fodder on two different textured calcareous soils. Soil Environ., 26(1): 33-41.

Romitti, M. V. da Silva, J. Gonzalez, A. Marolli, A. Arenhardt, E. Ghisleni, de Mamann, Â.T. Woschinski and B.L. Michel. 2016. The management of sowing density on yield and lodging in the main oat biotype grown in Brazil. Afr. J. Agric. Res., 11(21): 1935-1944. https:// doi.org/10.5897/AJAR2016.10909

Sarwar, M., M. Khan, Ajmal and Z. Iqbal. 2002. Status paper feed resources for livestock in Pakistan. Int. J. Agric. Biol., 4(1): 186-192.

Steel, R.G.D., J.H. Torrie and D.A. Dickey. 1997. Principles and procedures of statistics: A biometrical approach. WCB: McGraw-Hill, New York.

Yusuf, M.J., G. Nabi, A. Basit, S.K. Husnain and L.H. Akhtar. 2012. Development of high yielding millet variety "Sargodha Bajra-2011" released for general cultivation in Punjab Province of Pakistan. Pak. J. Agric. Sci., 49(3): 299-305. 